\title{
Assessing trustworthiness of system operability checking
}

\author{
A. Raza* and V. Ulansky** \\ *Projects and Maintenance Section, The Private Department of the President of the United Arab Emirates, P.O. Box: \\ 372, Abu Dhabi,UAE; ahmed_awan@hotmail.com \\ **Department of Electronics, Robotics, Monitoring and IoT Technologies, National Aviation University, 03058 Kyiv, \\ Ukraine; vladimir_ulansky@nau.edu.ua
}

\begin{abstract}
This study proposes a mathematical model for assessing the trustworthiness indicators of the operability checking for a deteriorating system. The set of mutually exclusive events at the time of operability checking are analyzed. Correct and incorrect decisions correspond to events such as true-positive, false-positive, true-negative, and false-negative. General expressions for computing the probabilities of possible decisions when checking the system operability at a discrete time are proposed. The paper introduces the effectiveness indicators of corrective maintenance such as average operating costs, total error probability, and a posteriori probability of failure-free operation. We illustrate the developed approach by calculating the probabilities of correct and incorrect decisions for a specific stochastic deterioration process.
\end{abstract}

\section{Introduction}

Maintenance based on equipment operability checking is widely used in systems with different physical properties. For commercial and military aircraft, such checks are performed after a predetermined time. Therefore, in the research literature, the mathematical maintenance models based on equipment operability checks are highly valued. Let us analyze some of the published studies relating to the corrective maintenance models with periodical or sequential checks. We consider studies that assume perfect repairs, i.e., after repairing, the system becomes as-good-as-new.

We can divide maintenance models based on operability checks on two groups. The checks or inspections are considered perfect in the first group, which means that correct decisions' conditional probabilities equal unity and incorrect decisions' conditional probabilities - zero. One of the first published studies in this area is Barlow et al. (1963). In this study, periodic and sequential plans of operability checks are considered. The checks are assumed to be perfect. The system failure can be detected only by inspection. The checking procedure's optimality criterion is the minimum total cost of checks and losses due to the system being in a state of unrevealed failure. Later, numerous studies were published on various cases related to this problem. For a review of studies in this research area, see Nakagawa (2005).

The checks are considered imperfect in the second group of corrective maintenance models, which means that correct decisions' conditional probabilities are less than unity and incorrect decisions' conditional probabilities are greater than zero. The correct decisions correspond to true-positive and true-negative events when checking the system operability. The incorrect decisions correspond to false-positive and falsenegative events. Morey (1967) published one of the first maintenance models with imperfect checks. In this study, the expected cost depends on the conditional probabilities of successfully detected failure $(p)$ and not detected failure $(1-p)$. The probabilities $p$ and $1-p$ correspond to conditional probabilities of true-negative and false-negative. However, the cost function does not include conditional probabilities of true-positive and false-positive. Kashtanov $(1981,1983)$ was perhaps the first to construct a cost function that considers all the conditional probabilities of correct and incorrect decisions when checking the system's operability. In addition to the probability $p$, Kashtanov introduced the conditional probability of a false-positive $q$ and truepositive $1-q$. Under the assumption that the probabilities $p$ and $q$ do not change over time, he considered the probabilities of multiple checks in the cost function. Further development of maintenance models based on operability checking with constant conditional probabilities of correct and incorrect decisions was carried out in the studies published by Berrade et al. (2013), Cavalcante et al. (2019), and many others. Ulansky (1987, 1988) proposed a generalized mathematical model of corrective maintenance based on operability checking. In this model, the conditional probabilities of correct and incorrect decisions depend on the system's operating time and the degradation process parameters.

Thus, we see that most of the published studies consider the probabilities of correct and incorrect decisions as constants, not depending on the time and degradation process parameters. 
This study proposes general equations for calculating the probabilities of true-positive, false-positive, true-negative, and false-negative. The events leading to correct and incorrect decisions are considered on the time axis, allowing incorporating these probabilities into the mathematical maintenance models.

\section{Decision rule at operability checking}

We consider a deteriorating system subjected to random failure whose condition is inspected at arbitrary time $t_{i}(i=1,2, \ldots)$. Let $S(t)$ be the system state parameter representing a non-stationary stochastic process with continuous time. Assume that between $S(t)$ and measurement error (or noise) $N(t)$ exists an additive relationship, i.e.,

$$
Y\left(t_{i}\right)=S\left(t_{i}\right)+N\left(t_{i}\right)
$$

where $Y\left(t_{i}\right)$ is the measurement result at time $t_{i}$.

When checking the system operability at time $t_{i}$, the following decision rule is used: if $y\left(t_{i}\right)<L$, the system is judged as operable at time $t_{i}$; if $y\left(t_{i}\right) \geq L$, the system is judged as inoperable, where $L$ is the functional failure threshold.

By the results of checking the system operability at time $t_{i}$, one of the following mutually exclusive events may appear:

$$
\begin{aligned}
& E_{1}\left(t_{i}\right)=\left\{S\left(t_{i}\right)<L \bigcap Y\left(t_{i}\right)<L\right\}, \\
& E_{2}\left(t_{i}\right)=\left\{S\left(t_{i}\right)<L \bigcap Y\left(t_{i}\right) \geq L\right\}, \\
& E_{3}\left(t_{i}\right)=\left\{S\left(t_{i}\right) \geq L \bigcap Y\left(t_{i}\right)<L\right\}, \\
& E_{4}\left(t_{i}\right)=\left\{S\left(t_{i}\right) \geq L \bigcap Y\left(t_{i}\right) \geq L\right\},
\end{aligned}
$$

where $E_{1}\left(t_{i}\right)$ is the joint occurrence of two events: the system is operable and judged as operable when checking at time $t_{i} ; E_{2}\left(t_{i}\right)$ is the joint occurrence of two events: the system is operable and judged as inoperable when checking at time $t_{i} ; E_{3}\left(t_{i}\right)$ is the joint occurrence of two events: the system is inoperable and judged as operable when checking at time $t_{i} ; E_{4}\left(t_{i}\right)$ is the joint occurrence of two events: the system is inoperable and judged as inoperable when checking at time $t_{i}$.

The events (2)-(5) are, respectively, called true-positive, false-positive, false-negative, and true-negative.

\section{Determination of trustworthiness indicators}

As can be seen in (2)-(5), the decision rule when checking system operability is based on the result of measurement of the system state parameter $S(t)$ at time $t_{i}$. Mathematically it means that in the space-time plane, we use vertical or space axis for decision making. Assuming that random variables $S\left(t_{i}\right)$ and $N\left(t_{i}\right)$ are independent and performing a few mathematical operations, we determine the probabilities of events (2)-(5) as follows:

$$
\begin{aligned}
& P\left[E_{1}\left(t_{i}\right)\right]=\int_{-\infty}^{L} \omega\left(s_{i}\right) \int_{-\infty}^{L-s_{i}} \theta\left(n_{i}\right) d n_{i} d s_{i}, \\
& P\left[E_{2}\left(t_{i}\right)\right]=\int_{-\infty}^{L} \omega\left(s_{i}\right) \int_{L-s_{i}}^{\infty} \theta\left(n_{i}\right) d n_{i} d s_{i}, \\
& P\left[E_{3}\left(t_{i}\right)\right]=\int_{L}^{\infty} \omega\left(s_{i}\right) \int_{-\infty}^{L-s_{i}} \theta\left(n_{i}\right) d n_{i} d s_{i}, \\
& P\left[E_{4}\left(t_{i}\right)\right]=\int_{L}^{\infty} \omega\left(s_{i}\right) \int_{L-s_{i}}^{\infty} \theta\left(n_{i}\right) d n_{i} d s_{i},
\end{aligned}
$$

where $\omega\left(s_{i}\right)$ is the probability density function (PDF) of random variable $S\left(t_{i}\right)$ and $\theta\left(n_{i}\right)$ is the PDF of random variable $N\left(t_{i}\right)$.

As we can see in (6)-(9), all limits in integrals are located on the space axis. Therefore, we cannot use these equations in solving reliability problems where the time axis is used.

This article proposes a method for calculating the probabilities $P\left[E_{1}\left(t_{i}\right)\right]-P\left[E_{4}\left(t_{i}\right)\right]$, in which events (2)-(5) are formulated in terms of time, making it possible to use the probabilities of correct and incorrect decisions in modeling reliability and maintenance problems.

Let $\mathrm{H}(\mathrm{H} \geq 0)$ be the random time to system failure with PDF $f(\eta)$. Let also introduce a new random variable $\mathrm{H}_{i}$ associated with the estimated time to failure based on the operability checking results at time $t_{i}$. 
We determine the random variables $\mathrm{H}$ and $\mathrm{H}_{i}$ as the smallest roots of the following stochastic equations:

$$
\begin{aligned}
& S(t)-L=0, \\
& Y\left(t_{i}\right)-L=0,
\end{aligned}
$$

From (11) follows that random variable $\mathrm{H}_{i}$ can be presented as follows:

$$
\mathrm{H}_{i}=\left\{\begin{array}{l}
t_{i}, \text { if } Y\left(t_{i}\right) \geq L(i=1,2, \ldots) \\
>t_{i}, \text { if } Y\left(t_{i}\right)<L
\end{array}\right\} .
$$

Using (12), we can convert the decision rule based on space (vertical) axis to the decision rule based on time (horizontal) axis: the system is judged as operable at time $t_{i}$ if $\eta_{i}>t_{i}$; otherwise (i.e., if $\eta_{i} \leq t_{i}$ ), the system is judged as inoperable, where $\eta_{i}$ is the realization of the random variable $\mathrm{H}_{i}$ for the system under inspection.

As it follows from (11), $\mathrm{H}_{i}$ is a function of random variables $\mathrm{H}$ and $N\left(t_{i}\right)$. Due to the presence of $N\left(t_{i}\right)$ in stochastic equation (11), a random error appears concerning the estimation of time to failure at time $t_{i}$, which is defined as follows:

$$
\Delta \mathrm{H}_{i}=\mathrm{H}_{i}-\mathrm{H}, i=1,2, \ldots
$$

As seen in (13), between random variables $\mathrm{H}(0<\mathrm{H}<\infty)$ and $\Delta \mathrm{H}_{i}\left(-\infty<\Delta \mathrm{H}_{i}<\infty\right)$ exists additive relationship. Therefore, random variable $\mathrm{H}_{i}$ varies from $-\infty$ to $\infty$. Due to a mismatch between the solutions of stochastic equations (10) and (11), one of the following mutually exclusive events can appear when checking the system operability at time $t_{i}$ :

$$
\begin{aligned}
& E_{1}\left(t_{i}\right)=\left\{\mathrm{H}>t_{i} \cap \mathrm{H}_{i}>t_{i}\right\}, \\
& E_{2}\left(t_{i}\right)=\left\{\mathrm{H}>t_{i} \cap \mathrm{H}_{i} \leq t_{i}\right\}, \\
& E_{3}\left(t_{i}\right)=\left\{\mathrm{H} \leq t_{i} \cap \mathrm{H}_{i}>t_{i}\right\}, \\
& E_{4}\left(t_{i}\right)=\left\{\mathrm{H} \leq t_{i} \cap \mathrm{H}_{i} \leq t_{i}\right\} .
\end{aligned}
$$

Determination of the probabilities of the events (14)-(17) reduces to the calculation of the probability that the random point $\left(\mathrm{H}, \mathrm{H}_{i}\right)$ falls into the 2-dimensional region formed by the limits of the variation of each random variable, and is equal to 2 -fold integral over this region.

Let $f_{0}\left(\eta, \eta_{i}\right)$ be the joint PDF of random variables $\left(\mathrm{H}, \mathrm{H}_{i}\right)$. The event $E_{1}\left(t_{i}\right)$ corresponds to a 2-dimensional region with the following limits: $t_{i} \leq \mathrm{H}<\infty$ and $t_{i}<\mathrm{H}_{i}<\infty$. By integrating the PDF $f_{0}\left(\eta, \eta_{i}\right)$ within the specified region, we determine the probability of the event $E_{1}\left(t_{i}\right)$

$$
P\left[E_{1}\left(t_{i}\right)\right]=\int_{t_{i}}^{\infty} \int_{t_{i}}^{\infty} f_{0}\left(\eta, \eta_{i}\right) d \eta_{i} d \eta
$$

The event $E_{2}\left(t_{i}\right)$ corresponds to a 2-dimensional region with the following limits: $t_{i} \leq \mathrm{H}<\infty$ and $-\infty<\mathrm{H}_{i}$ $\leq t_{i}$. By integrating the PDF $f_{0}\left(\eta, \eta_{i}\right)$ within the specified region, we determine the probability of the event $E_{2}\left(t_{i}\right)$

$$
P\left[E_{2}\left(t_{i}\right)\right]=\int_{t_{i}}^{\infty} \int_{-\infty}^{t_{i}} f_{0}\left(\eta, \eta_{i}\right) d \eta_{i} d \eta
$$

The event $E_{3}\left(t_{i}\right)$ corresponds to a 2-dimensional region with the following limits: $-\infty<\mathrm{H} \leq t_{i}$ and $t_{i} \leq \mathrm{H}_{i}<$ $\infty$. By integrating the PDF $f_{0}\left(\eta, \eta_{i}\right)$ within the specified region, we determine the probability of the event $E_{3}\left(t_{i}\right)$

$$
P\left[E_{3}\left(t_{i}\right)\right]=\int_{-\infty}^{t_{i}} \int_{t_{i}}^{\infty} f_{0}\left(\eta, \eta_{i}\right) d \eta_{i} d \eta
$$

The event $E_{4}\left(t_{i}\right)$ corresponds to a 2-dimensional region with the following limits: $-\infty<\mathrm{H} \leq t_{i}$ and $-\infty<$ $\mathrm{H}_{i} \leq t_{i}$. By integrating the $\operatorname{PDF} f_{0}\left(\eta, \eta_{i}\right)$ within the specified region, we determine the probability of the event $E_{4}\left(t_{i}\right)$

$$
P\left[E_{4}\left(t_{i}\right)\right]=\int_{-\infty}^{t_{i}} \int_{-\infty}^{t_{i}} f_{0}\left(\eta, \eta_{i}\right) d \eta_{i} d \eta
$$


As we can see in (18)-(21), all limits in integrals are located on the time axis. Therefore, one can incorporate the probabilities (18)-(21) into the reliability and maintenance models.

As seen from (18)-(21), to find the probabilities of true-positive, false-positive, false-negative, and truenegative when checking system operability, the joint $\mathrm{PDF} f_{0}\left(\eta, \eta_{i}\right)$ of random variables $\left(\mathrm{H}, \mathrm{H}_{i}\right)$ must be known. Following the multiplication theorem of PDFs, we represent the PDF $f_{0}\left(\eta, \eta_{i}\right)$ in the following form:

$$
f_{0}\left(\eta, \eta_{i}\right)=f(\eta) \psi\left(\eta_{i} \mid \eta\right)
$$

where $\psi\left(\eta_{i} \mid \eta\right)$ is the conditional PDF of random variable $\mathrm{H}_{i}$ provided that $\mathrm{H}=\eta$.

In the case of $\mathrm{H}=\eta$, the random variable $\mathrm{H}_{i}$ is presented as $\mathrm{H}_{i}=\eta+\Delta \mathrm{H}_{i}$. Due to the additive relationship between random variables $\mathrm{H}$ and $\Delta \mathrm{H}_{i}$, the following equality holds:

$$
\psi\left(\eta_{i} \mid \eta\right)=\psi_{0}\left(\delta_{i} \mid \eta\right)=\psi_{0}\left(\eta_{i}-\eta \mid \eta\right),
$$

where $\psi_{0}\left(\delta_{i} \mid \eta\right)$ is the conditional $\mathrm{PDF}$ of random variable $\Delta \mathrm{H}_{i}$ provided that $\mathrm{H}=\eta$.

By substitution of (23) into (22), we obtain

$$
f_{0}\left(\eta, \eta_{i}\right)=f(\eta) \psi_{0}\left(\eta_{i}-\eta \mid \eta\right) .
$$

The relation (24) makes it possible to simplify (18)-(21). We obtain the probability of true-positive by substituting (24) into (18)

$$
P_{T P}\left(t_{i}\right)=P\left[E_{1}\left(t_{i}\right)\right]=\int_{t_{i}}^{\infty} f(\eta) \int_{t_{i}}^{\infty} \psi_{0}\left(\eta_{i}-\eta \mid \eta\right) d \eta_{i} d \eta .
$$

Assuming that $\delta_{i}=\eta_{i}-\eta$ in (25), we obtain

$$
P_{T P}\left(t_{i}\right)=\int_{t_{i}}^{\infty} f(\eta) \int_{t_{i}-\eta}^{\infty} \psi_{0}\left(\delta_{i} \mid \eta\right) d \delta_{i} d \eta .
$$

By performing analogous changes of variables in (19)-(21), we obtain the following:

- the probability of false-positive

$$
P_{F P}\left(t_{i}\right)=P\left[E_{2}\left(t_{i}\right)\right]=\int_{t_{i}}^{\infty} f(\eta) \int_{-\infty}^{t_{i}-\eta} \psi_{0}\left(\delta_{i} \mid \eta\right) d \delta_{i} d \eta,
$$

- the probability of false-negative

$$
P_{F N}\left(t_{i}\right)=P\left[E_{3}\left(t_{i}\right)\right]=\int_{0}^{t_{i}} f(\eta) \int_{t_{i}-\eta}^{\infty} \psi_{0}\left(\delta_{i} \mid \eta\right) d \delta_{i} d \eta,
$$

- the probability of true-negative

$$
P_{T N}\left(t_{i}\right)=P\left[E_{4}\left(t_{i}\right)\right]=\int_{0}^{t_{i}} f(\eta) \int_{-\infty}^{t_{i}-\eta} \psi_{0}\left(\delta_{i} \mid \eta\right) d \delta_{i} d \eta .
$$

In modeling reliability and maintenance problems, it is often necessary to know the conditional probabilities of true-positive, false-positive, false-negative, and true-negative given that $\mathrm{H}=\eta$. We can find these probabilities from (26)-(29) as follows:

$$
\begin{aligned}
& P_{T P}\left(t_{i} \mid \eta\right)=\int_{t_{i}-\eta}^{\infty} \psi_{0}\left(\delta_{i} \mid \eta\right) d \delta_{i}\left(t_{i} \leq \eta<\infty\right), \\
& P_{F P}\left(t_{i} \mid \eta\right)=\int_{-\infty}^{t_{i}-\eta} \psi_{0}\left(\delta_{i} \mid \eta\right) d \delta_{i}\left(t_{i} \leq \eta<\infty\right), \\
& P_{F N}\left(t_{i} \mid \eta\right)=\int_{t_{i}-\eta}^{\infty} \psi_{0}\left(\delta_{i} \mid \eta\right) d \delta_{i}\left(-\infty<\eta \leq t_{i}\right), \\
& P_{T N}\left(t_{i} \mid \eta\right)=\int_{-\infty}^{t_{i}-\eta} \psi_{0}\left(\delta_{i} \mid \eta\right) d \delta_{i}\left(-\infty<\eta \leq t_{i}\right) .
\end{aligned}
$$

Figure 1 shows a graphical presentation of conditional probabilities of true-positive and false-positive (a) and true-negative and false-negative (b). 


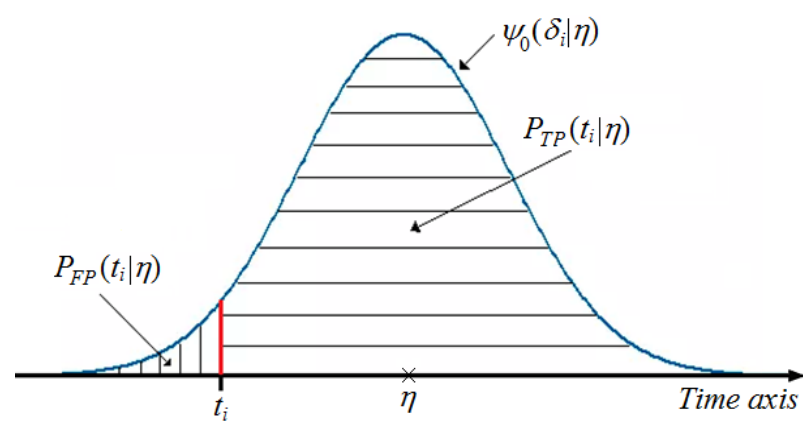

(a)

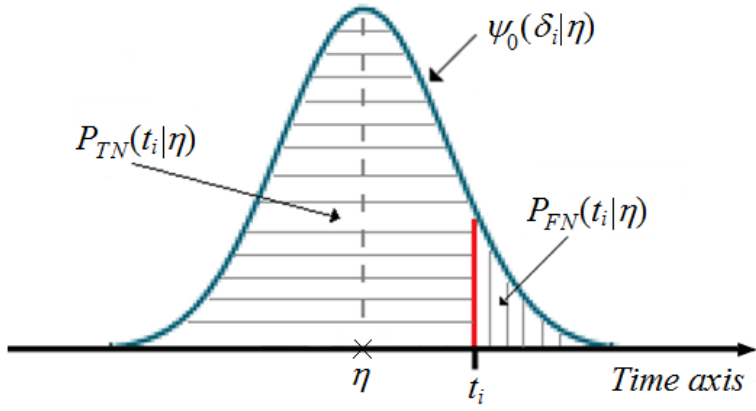

(b)

Figure 1. Graphical presentation of conditional probabilities of true-positive and false-positive (a) and truenegative and false-negative (b)

\section{Effectiveness indicators of corrective maintenance}

We determine the effectiveness indicators of corrective maintenance at time $t_{i}$ as follows:

- the average maintenance cost is given by

$$
C_{C M}\left(t_{i}\right)=\left(C_{P R}+C_{S P}\right) P_{F P}\left(t_{i}\right)+C_{U F} P_{F N}\left(t_{i}\right)+C_{C R} P_{T N}\left(t_{i}\right),
$$

- the total error probability

$$
P_{\text {ERROR }}\left(t_{i}\right)=P_{F P}\left(t_{i}\right)+P_{F N}\left(t_{i}\right),
$$

- the posterior probability of the system operability

$$
P\left\{\mathrm{H}>t_{i} \mid \mathrm{H}_{i}>t_{i}\right\}=\frac{P_{T P}\left(t_{i}\right)}{P_{T P}\left(t_{i}\right)+P_{F N}\left(t_{i}\right)},
$$

where $C_{P R}$ is the average cost of preventive repair of the system due to false-positive, $C_{S P}$ is the average cost of additional spare parts due to untimely preventive repair of the system, $C_{C R}$ is the average cost of corrective repair of the system, and $C_{U F}$ is the average loss due to missed failure detection when checking the system operability.

\section{Modeling the deterioration process}

Monotone stochastic functions can describe many deterioration processes. Examples of such processes are cracks in wind turbine rotor blades (Valeti \& Pakzad, 2018) and concrete and metal constructions (Shaikh, 2018), power in radars with active phased antenna arrays (Kostanovsky et al., 2019), and many others.

Suppose that the following monotonic random process describes the state of the system:

$$
S(t)=S_{0}+S_{1} t^{\gamma},
$$

where $S_{0}$ is the initial random value of the system state parameter $S(t)$, defined in the range from 0 to $L, S_{1}$ is the random degradation rate of the system state parameter, defined in the interval from 0 to $\infty$, and $\gamma$ is the exponent of time.

Using method for determining PDF $\psi_{0}\left(\delta_{i} \mid \eta\right)$ given in (Raza \& Ulansky, 2017), we derive the following equation:

$$
\psi_{0}\left(\delta_{i} \mid \eta\right)=\gamma \int_{0}^{L} \rho\left(s_{0}\right) f\left(\eta \mid s_{0}\right)\left(\frac{L-s_{0}}{\eta}\right)\left|\left(\frac{\delta_{i}+\eta}{\eta}\right)^{\gamma-1}\right| \varphi\left\{\left(L-s_{0}\right)\left[1-\left(\frac{\delta_{i}+\eta}{\eta}\right)^{\gamma}\right]\right\} \frac{d s_{0}}{f(\eta)},
$$

where $\rho\left(s_{0}\right)$ is the PDF of random variable $S_{0}, f\left(\eta \mid s_{0}\right)$ is the conditional PDF of random variable $\mathrm{H}$ provided that $S_{0}=s_{0}$, and $\varphi\left(n_{i}\right)$ is the PDF of the random measurement error at time $t_{i}$.

If the initial value of the system state parameter is almost identical for different systems, i. e., $S_{0}=s_{0}=$ constant, then (38) converts to the following PDF:

$$
\psi_{0}\left(\delta_{i} \mid \eta\right)=\gamma\left(\frac{L-s_{0}}{\eta}\right)\left|\left(\frac{\delta_{i}+\eta}{\eta}\right)^{\gamma-1}\right| \varphi\left\{\left(L-s_{0}\right)\left[1-\left(\frac{\delta_{i}+\eta}{\eta}\right)^{\gamma}\right]\right\} .
$$


If the measurement error $N\left(t_{i}\right)$ has normal distribution with zero mathematical expectation and standard deviation $\sigma_{n}$, we obtain from (39) that

$$
\psi_{0}\left(\delta_{i} \mid \eta\right)=\left(\frac{\gamma}{\sigma_{n} \sqrt{2 \pi}}\right)\left(\frac{L-s_{0}}{\eta}\right)\left|\left(\frac{\delta_{i}+\eta}{\eta}\right)^{\gamma-1}\right| \exp \left\{-\frac{1}{2 \sigma_{n}^{2}}\left[\left(L-s_{0}\right)\left[1-\left(\frac{\delta_{i}+\eta}{\eta}\right)^{\gamma}\right]\right]^{2}\right] .
$$

\section{Results and discussion}

Let $S(t)$ be the power supply voltage of the radar transmitter and $N\left(t_{i}\right)$ has a normal distribution with zero mathematical expectation and standard deviation $\sigma_{n}$. According to (Ma, 2013), the radar transmitter is operable if $S(t) \leq 25 \mathrm{kV}$. Therefore, the threshold $L$ is $25 \mathrm{kV}$. Let $m_{S 1}=0.01 \mathrm{kV} / \mathrm{h}, \sigma_{S 1}=0.0043 \mathrm{kV} / \mathrm{h}, \gamma=0.9$, $s_{0}=19.92 \mathrm{kV}$, and $\sigma_{n}=0.5 \mathrm{kV}$, where $m_{S 1}$ and $\sigma_{S 1}$ are, respectively, the mathematical expectation and standard deviation of random variable $S_{1}$.

Figure 2 shows the dependency of true-positive (a) and false-positive (b) versus time. As we can see in Fig. 2 (a), the probability of true-positive monotonically decreases with time, reaching $15 \%$ at $t=2000 \mathrm{~h}$. The behavior of $P_{T P}(t)$ is similar to the reliability function but the latter has higher values. As shown in Fig. 2 (b), the probability of false-positive is almost zero till time $t=325 \mathrm{~h}$; then, when $t>325 \mathrm{~h}$, this probability increases reaching a maximum of $4.3 \%$ at $t=800 \mathrm{~h}$, and when $t>800 \mathrm{~h}$ it starts to decrease reaching $1 \%$ at $t=2000 \mathrm{~h}$.

Figure 3 shows the dependency of true-negative (a) and false-negative (b) versus time. As shown in Fig. 3 (a), the probability of true-negative increases with time, reaching $85 \%$ at $t=2000 \mathrm{~h}$. The shape of $P_{T N}(t)$ is similar to that of the cumulative distribution function of time to failure but the latter has higher values.

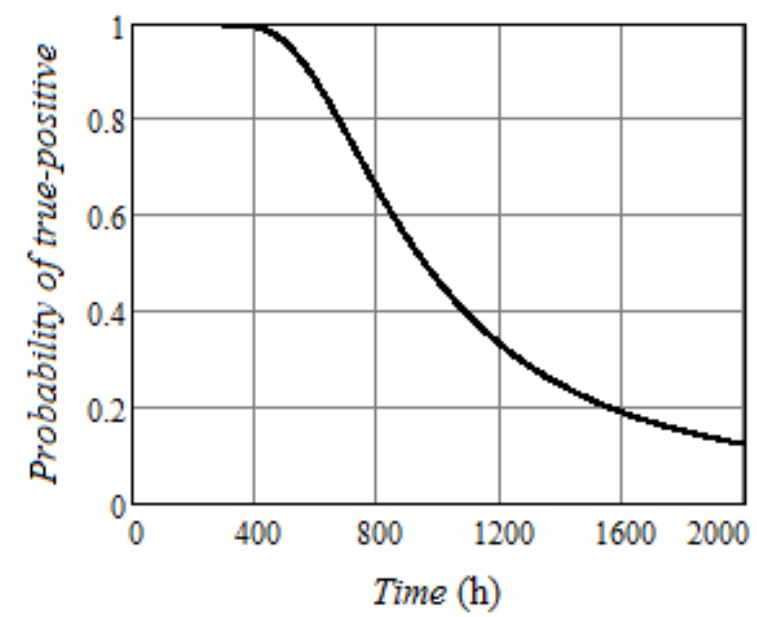

(a)

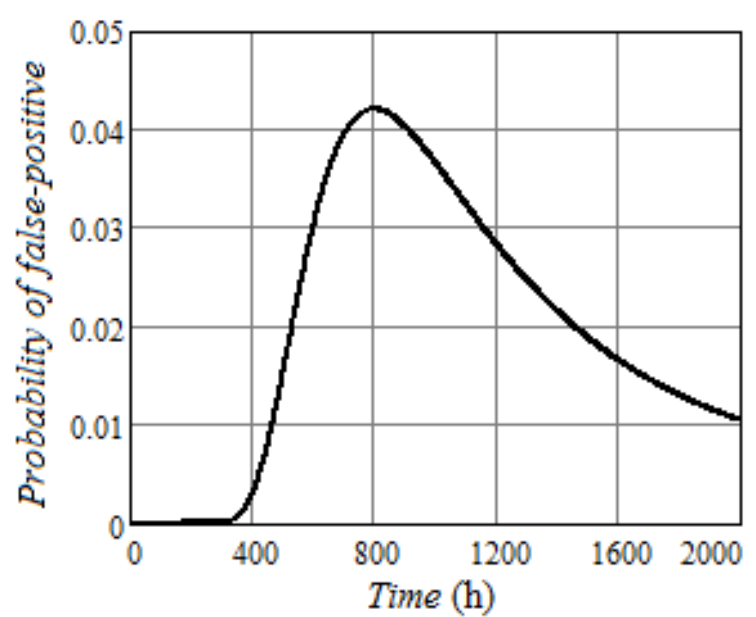

(b)

Figure 2. The dependency of true-positive (a) and false-positive (b) versus time

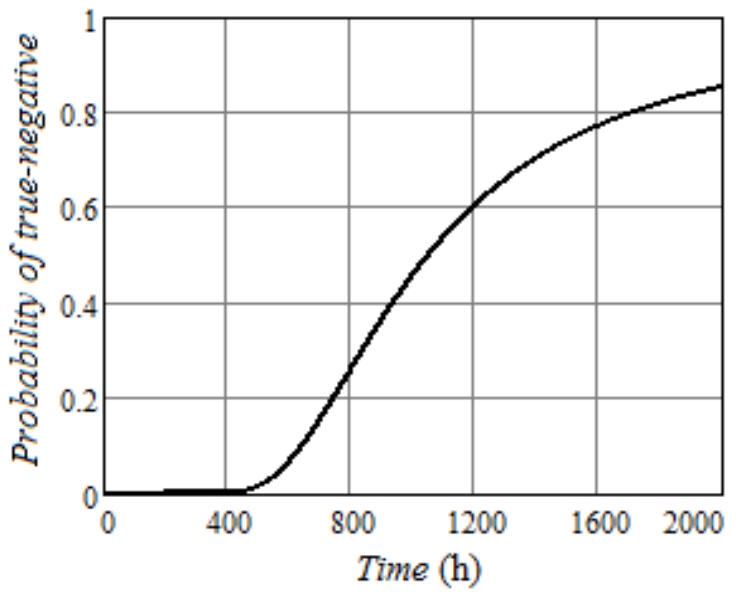

(a)

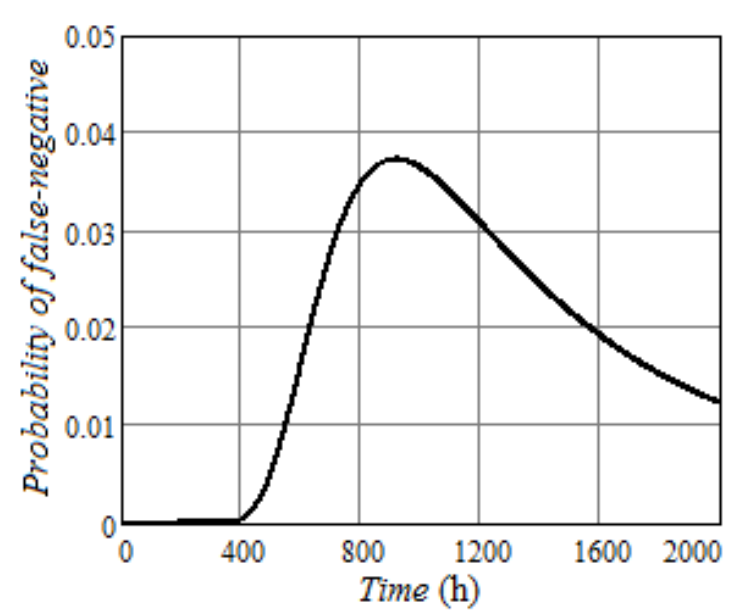

(b)

Figure 3. The dependency of true-negative (a) and false-negative (b) versus time 
As we can see in Fig. 3 (b), the probability of false-negative is almost zero till time $t=400 \mathrm{~h}$; then, when $t$ $>400 \mathrm{~h}$, this probability increases reaching a maximum of $3.8 \%$ at $t=950 \mathrm{~h}$, and when $t>950 \mathrm{~h}$ it decreases reaching $1.2 \%$ at $t=2000 \mathrm{~h}$.

Thus, Figures 2 and 3 confirm that the probabilities of correct and incorrect decisions when checking the system operability are functions of time and can't be considered as constants.

\section{Conclusion}

The main contribution of this article is to show how the probabilities of true-positive, false-positive, truenegative, and false-negative are determined when checking the system operability for the case when events are expressed in terms of time. Contrary to previous studies, the decision rule when checking the system's operability was introduced on the time axis. We used the stochastic equations of the first hitting time for the degradation process and the sum of the degradation process and measurement noise to formulate events leading to correct and incorrect decisions. General expressions for the probabilities of true-positive, falsepositive, true-negative, and false-negative have been derived, which are applicable for an arbitrary degradation process and measurement noise. We presented equations for such indicators of the corrective maintenance effectiveness as the average cost of maintenance, the total error probability, and the posterior probability of operability, including the probabilities of correct and incorrect decisions. For a nonlinear stochastic process of degradation with monotonic realizations, we derived the conditional PDF of the error in evaluating the operating time to failure through the known PDF of the system state parameter's measurement error and the value of the functional failure threshold. A numerical example illustrated that probabilities of correct and incorrect decisions when checking the system's operability are very much functions of time despite the large number of published studies where these probabilities are considered independent of time.

\section{References}

Barlow RE, Hunter LC, Proshan H (1963) Optimum checking procedures. J. Soc. Industrial and Appl. Math. 11(4), 1078-1095.

Berrade MD, Cavalcante CAV, Scarf PA (2013) Modelling imperfect inspection over a finite horizon. Reliability Engineering and System Safety 111, 18-29.

Cavalcante CAV, Scarf PA, Berrade MD (2019) Imperfect inspection with unrevealed failure and an unrevealed defective state. IEEE Transactions on Reliability 68(2), 764-775.

Güemes A, Fernández-López A, Díaz-Maroto PF (2018) Structural health monitoring in composite structures by fiber-optic sensors. Sensors 18(4), 1-11.

Kashtanov VA (1981) Optimum Problems of Maintenance. Znanie. (in Russian)

Kashtanov VA (1983) Maintenance models with limited reliability information and checking errors. In: Questions of the Mathematical Theory of Reliability. Ed. Gnedenko BV. Radio i Svyaz. (in Russian)

Kostanovsky V, Machalin IA, Kozachuk O, Terentyeva I (2019) Construction of a generalized probabilisticphysical model of reliability of a two-level active phased antenna array. Eastern-European Journal of Enterprise Technologies 3(9), 31-40.

Ma C, Shao Y, Ma R (2013) Analysis of equipment fault prediction based on metabolism combined model. Journal of Machinery Manufacturing and Automation 2(3), 58-62.

Morey RC (1967) A criterion for the economic application of imperfect inspections. Operations Research 15, 695-698.

Nakagawa T (2005). Maintenance Theory of Reliability. Springer.

Raza A, Ulansky V (2017) Modelling of predictive maintenance for a periodically inspected system. Procedia CIRP 59, 95-101.

Shaikh FUA (2018) Effect of cracking on corrosion of steel in concrete. International Journal of Concrete Structures and Materials 12, 1-12.

Ulansky VV (1987) Optimal maintenance plans for radio-electronic systems based on diagnostics. Issues of Technical Diagnostics. (in Russian)

Ulansky VV (1988) Diagnostic support of aircraft radio-electronic systems operation. D.Sc. thesis. National Aviation University (in Russian).

Valeti B, Pakzad SN (2018) Remaining Useful Life Estimation of Wind Turbine Blades under Variable Wind Speed Conditions using Particle Filters. In Proc.: Annual Conference on the Prognostics and Health Management Society, pp. 1-10. 